\title{
Ultrasound Evaluation of Breast Masses and Histopathology Correlation
}

\author{
B. Vinod Kumar ${ }^{1}$, A. Ravi Kumar ${ }^{2}$ \\ ${ }^{1}$ Professor and H.O.D., ${ }^{2}$ Associate Professor, Department of Radiology, Fathima Institute Of Medical Sciences, Kadapa, \\ Andhra Pradesh, India.
}

Corresponding author: Dr B. Vinod Kumar, Professor and H.O.D, Department of Radiology, Fathima Institute Of Medical Sciences, Kadapa, Andhra Pradesh, India

DOI: 10.21276/ijcmsr.2018.3.2.21

How to cite this article: B. Vinod Kumar, A. Ravi Kumar. Ultrasound evaluation of breast masses and histopathology correlation. International Journal of Contemporary Medicine Surgery and Radiology. 2018;3(2):B85-B88.

\section{A B S T R A C T}

Introdution: Breast ultrasonography (US) has gained acceptance as a diagnostic tool for the evaluation of breast diseases that are obscured by dense breast tissue in mammography can be detected with Ultrasonogram. Study aimed to evaluate the correlation of the US findings including shape, boundary zone, internal and posterior echo, anterior and posterior borders, estimated histological types and carcinoma infiltration with their corresponding histopathological findings of the breast lesions.

Material and methods: This was a hospital-based retrospective study which was carried out at department of radiology, Fathima Institute of Medical Sciences, Kadapa, Andhra Pradesh, during the period from March 2016 to December 2017. A sample size of 50 breast lesions was used for analysis and the protocol was approved by ethics committee.

Results: The overall detection rate of carcinoma extension by US was $86 \%$ ( 43 out of the 50 tumors). 5 out of the 7 tumors $(71.4 \%)$ in which US could not detect carcinoma extension demonstrated low-grade intraductal components, and these lesions were also, $1 \mathrm{~mm}$ in diameter. 1 out of these 7 cases turned out to be invasive ILC in which carcinoma cells invaded with forming single cell pattern and small LCIS extension.

Conclusion: US cannot define the extension of carcinoma preoperatively, correlating US findings with histopathologic features of carcinoma cells can determine the extension of carcinoma.

Key words: Ultrasonography (US), Histopathological findings of the breast lesions, Ductal Carcinoma in Situ (DCIS), Invasive Lobular Carcinoma (ILC).

\section{INTRODUCTION}

Breast cancer is the more common cancer observed in women worldwide representing nearly a quarter $(25 \%)$ of all cancers. Women from less developed regions (883 000 cases) have slightly more number of cases compared to more developed (794 000) regions. ${ }^{1}$

In India, although age adjusted incidence rate of breast cancer is lower (25.8 per 100 000) than United Kingdom (95 per 100 000) but mortality is at par (12.7 vs 17.1 per 100 000) with United Kingdom. ${ }^{2}$ There is a significant increased incidence and cancer-associated morbidity and mortality in Indian subcontinent as described in global and Indian studies..$^{3-7}$ Currently the incidence of breast cancer has surpassed cervical cancer and is leading cause of cancer death among Indian women. ${ }^{8}$

Epidemiology of breast cancer across different PBCRs in India shows increasing trends for incidence and mortality mainly due to rapid urbanization, industrialization, population growth and ageing affecting almost all parts of India. Marital status, location (urban/rural), BMI, breast feeding, waist to hip ratio, obesity, alcohol consumption, tobacco chewing, smoking, lack of exercise, diet, environmental factors were major risk factors in India leading to increasing incidence of cancer. Mortality rate in India is on rise due to late diagnosis because of lack of awareness and financial constrains in some regions. A multidisciplinary approach to breast cancer including awareness programs, preventive measure, screening programs for early detection and availability of treatment facilities are vital for reducing both incidence and mortality of breast cancer in Indian women. Breast cancer projection for India during time periods 2020 suggests the number to go as high as $17,97,900$ with its approximately relative percentage remaining same $10 \%$ among all the cancers. ${ }^{8}$ Appearance of breast carcinoma vary greatly in mammograohy. ${ }^{9}$ At present breast ultrasonography (US) has gained widespread acceptance as a diagnostic tool for the evaluation of breast disorders. ${ }^{10}$ Breast diseases that are obscured by dense breast tissue in mammography can be detected with US. US serves better in the detection of breast cancer if the patient is young or the masses are small. ${ }^{11,12}$

Previous studies have demonstrated the diagnostic benefits of US in differentiating benign from malignant breast disease. Carcinomas are classically described as irregular solid masses with a heterogenous texture and reduced sound transmission in the US, resulting in 'shadowing' behind the lesion. Not all 
carcinomas fulfill these criteria and some do only partially. In general, an accurate correlation of US findings with their corresponding histopathologic features is considered most important in US evaluation in this setting. ${ }^{13}$

Breast-conserving surgery is most common treatment of early breast cancer in India. Before performing conserving surgery, it is very important to detect carcinoma extension and determine the excision area as accurately as possible for the benefits of the patients. ${ }^{13}$

US can detect smaller non-palpable cancers by using high resolution equipment which are not generally detected on high-quality mammography. However, few studies have demonstrated the limitation of the US to detect small lesions. ${ }^{13}$ Therefore, we tried to evaluate breast masses in our hospital set up using US and correlate US findings with histopathologic features of carcinoma cells.

Materials and Methods:

This was a hospital-based retrospective study which was carried out at department of radiology, Fathima Institute of Medical Sciences, Kadapa, Andhra Pradesh, during the period from March 2016 to December 2017. A sample size of 50 breast lesions was used for analysis and the protocol was approved by ethical committee.

\section{Inclusion criteria}

Breast lesions which were initially detected by US during the time period from March 2016 to December 2017 were included in the study.

\section{Exclusion criteria}

The cases treated with neo-adjuvant chemotherapy were excluded from the study.

\section{US and histopathologic analyses}

The Ultrsound examination carried out using the mechanical scanners as mechanical scanners 5 Siemens sonoline G50 (Siemens USA) with a $10-\mathrm{MHz}$ transducer, in department of Radiology, Fathima Institute of Medical Sciences, Kadapa, Andhra Pradesh

Surgical specimens fixed in 10\% formaldehyde solution and then cut into serial 5-mm thick slices. Histopathological slides of each tumor independently without knowledge of the breast US findings two pathologists opinion was obtained. They used Olympus (Tokyo, Japan) BX50 and 20X objectives for the analysis.

Malignant cells at the surgical margin and within $5 \mathrm{~mm}$ of the surgical margin it was defined as positive margin. The accurate ratio between the cancer extension detected by the US and the histopathologic cancer extension was evaluated. If there were discrepancies of carcinoma extension and estimated histological types among these modalities, we returned to examine the discrepant lesions by US again.

\section{STATISTICAL ANALYSIS}

Collected data were compiled, managed, analysed and presented using Statistical Package for Social Sciences (SPSS) software and MS Excel.

\section{RESULTS}

\section{Evaluation of The Margins of the Lesion}

Nine out of the 50 were circumscribed masses. Of the 9 circumscribed tumors detected by US, 7 cases (77.8\%) were also histopathologically circumscribed. Not circumscribed masses were 41 tumors in our present study. Thirty seven out of these 41 tumors (90.25\%) were also histopathologically 'not circumscribed'. The rate of concordance between US and histopathological findings was $87.0 \%$. (Table-2)

\section{Boundary Zone (Halo)}

29 out of these 50 tumors were recognized with halo using US. 26 out of these 29 tumors with halo were defined as 'histopathologic halo' (Table 2). The rate of concordance was $87.6 \%$. 21 out of the 50 tumors turned out to be the masses without halo. (Table-2)

\section{Internal And Posterior Echoes}

About $59.7 \%$ of the tumors in which internal echoes were equal / heterogeneous histopathologically were associated with poor collagenized stroma and heterogenous intratumoral structure (the ratio of carcinoma cells to stroma was 3:1-1:3), tumors associated with low echo levels demonstrated marked collagenized stroma and the higher fibroblastic stromal ratio. As for posterior echo, accentuating tumors histopathologically demonstrated carcinoma cells proliferated in pushing, encapsuled and monotonous fashions, and were also demonstrated in all mucinous carcinoma examined. About $76.1 \%$ of the tumors classified as 'no changes' also demonstrated the patterns of marked intratumoral heterogeneity. In addition, ulrtasonographically

\begin{tabular}{|l|c|}
\hline Histological types (all) & $\mathbf{5 0}$ \\
\hline Invasive ductal carcinoma (IDC) & 43 \\
\hline Ductal carcinoma in situ (DCIS) & 2 \\
\hline Invasive lobular carcinoma (ILC) & 3 \\
\hline Mucinous carcinoma & 2 \\
\hline \multicolumn{2}{|c|}{ Table-1: Histological types } \\
\hline
\end{tabular}

\begin{tabular}{|l|c|c|}
\hline US findings & $\begin{array}{c}\text { No. } \\
\text { of } \\
\text { cases }\end{array}$ & $\begin{array}{c}\text { Rate of } \\
\text { concordance } \\
\text { (\%) }\end{array}$ \\
\hline Margin \\
\hline Circumscribed \\
\hline Non Circumscribed & 9 & 77.8 \\
\hline Boundary zone (halo) & 41 & 90.25 \\
\hline Halo (+) & 29 & 87.6 \\
\hline Halo (-) & 21 & 86.2 \\
\hline Associated findings (Interruption of the mammary borders) \\
\hline Interruption & 36 & 83.4 \\
\hline Non- Interruption & 14 & 85.7 \\
\hline \multicolumn{3}{|c|}{ Table-2: US findings } \\
\hline
\end{tabular}

\begin{tabular}{|l|l|l|l|}
\hline $\begin{array}{l}\text { Histological } \\
\text { types }\end{array}$ & Concordance & $\begin{array}{l}\text { Not } \\
\text { concordance }\end{array}$ & $\begin{array}{l}\text { Rate of } \\
\text { concordance } \\
\text { (\%) }\end{array}$ \\
\hline IDC & 39 & 2 & 95.2 \\
\hline DCIS & 1 & 3 & 25 \\
\hline ILC & 4 & 6 & 66.7 \\
\hline Mucinous & 1 & 1 & 100 \\
\hline \multicolumn{3}{|c|}{ Table-3: The concordance rates between US diagnosis and the } \\
\hline
\end{tabular}


attenuating cases (14 out of the 50 tumors, 28\%) were associated with marked collagenized stroma and higher fibroblastic stromal ratio. (Figure-1)

\section{Histopathological Correlations With Other Ultrasonographic Findings (Interruption Of The} Anterior Or Posterior Borders OfThe Mammary Gland) Interruption of anterior and posterior borders tumors were detected in 36 out of the 50 tumors. 30 out of the 36 tumors (83.4\%) were also histopathologically interpreted as extension into adipose tissue. Non-interruption tumors were seen in 14 cases. 12 out of the 14 tumors (85.7\%) were histopathologically infiltration in mammary gland or noninvasive carcinomas. The rate of concordance of these borders was $84.4 \%$. (Table-2)

\section{The Correlation Between Final Ultrasound And Histopathological Diagnoses}

The ratio of the correlation between histological types by Ultrasound diagnosis and histopathological types was $91.6 \%$ (46 out of the 50 tumors). The concordance rates between US findings and the following histologic types; IDC, DCIS, ILC and mucinous carcinoma were $95.2 \%$ (39 out of the 41 tumors), 25\% (1 out of the 4 tumors), $66.7 \%$ (4 out of the 6 tumors) and $100.0 \%$ ( 1 out of 1 tumor), respectively (Table 3). Ultrasound has limited in its ability to detect the lesions with, $1 \mathrm{~mm}$ in diameter.

\section{Correlation Between US and Histopathological Carcinoma Extension:}

The overall detection rate of carcinoma extension by Ultrasonography was $86 \%$ (43 out of the 50 tumors). 5 out of the 7 tumors (71.4\%) in which US could not detect carcinoma extension demonstrated low-grade intraductal components, and these lesions were also, $1 \mathrm{~mm}$ in diameter. 1 out of these 7 cases turned out to be invasive ILC in which carcinoma cells invaded with forming single cell pattern and small LCIS extension. One was the infiltrated lesion of IDC. This tumor was invaded with forming single cell pattern with poor stromal reaction, similar to ILC.

\section{DISCUSSION}

Circumscribed masses are usually detected in cases in which carcinoma cells proliferated in both solid and expanded fashion. On the other side, non-circumscribed masses are detected when cells are arranged in clusters, cords and trabeculae and associated with mixed intraductal component and invasive areas. Halo is generally defined as one of back scattering in US. ${ }^{15}$ Such opposite sound wave of incident element is generally defined as back scattering. ${ }^{16}$ Results of our present study demonstrated that halo was indeed characterized by the following histopathologic features, carcinoma cells infiltrated or mixed fat tissue, carcinoma cells and fibroblastic stroma.

Literature suggests that the degree of internal hypoechogenicity determines its sensitivity in predicting malignancy of the lesion. ${ }^{17,18,19}$ And also, posterior shadowing has been also reported as one of the important US features suggestive of malignant nature of the lesions. ${ }^{17,18,20}$ Our study demonstrated that anterior and posterior echoes were caused by the ratio of intratumoral carcinoma cells and fibroblastic stroma, and histological stromal characteristics. And also results of our present study demonstrated that internal echoes and posterior echoes were defined by histopathological intratumoral construction. Internal and posterior acoustic shadowing is considered the direct result of US beam attenuation by the desmoplastic reaction to breast cancer. ${ }^{21}$ Our study demonstrated that internal low echoic masses represented the high ratio of fibroblastic stroma and the stroma in these lesions turned out to have marked degrees of interstitial collagenization. And also, attenuation of posterior echo was detected in the tumors histopathologically associated with hyperplasia of collagenized fibroblastic stroma.

This study show that the concordance rate between the US findings and the histopathology findings was $91.6 \%$, now it could reasonably postulate that histological types from the US findings. Results from this study had shown that DCIS and ILC were having lower concordance rates between estimated and actual histological types than the other types. This study clearly demonstrated the detection rate of carcinoma extension was $86 \%$ by US. Tumors where US cannot appreciate carcinoma extension corresponded to DCIS confirmed histopathological evaluations. ILC can be insidious and difficult to detect on routine physical examination and/or imaging including US. In addition, our study demonstrated that US is limited to detect the lesions with less than $1 \mathrm{~mm}$ in diameter.

\section{CONCLUSION}

Breast conservation therapy is treatment standard for the great majority of breast carcinoma. Several investigations reported the association of higher tumor recurrence rates with positive or close margins than with negative margins following breast-conserving therapy. so it becomes very important to evaluate carcinoma extension preoperatively and determine the excision areas for performing breastconserving surgery as accurate as possible. Results of this study we conclude that cases where US cannot define the extension of carcinoma preoperatively, correlating US findings with histopathologic features of carcinoma cells can determine the extension of carcinoma. Study demonstrates the importance of correlation between histopathological and ultrasonographic findings of the breast lesions for improving the quality of US.

\section{REFERENCES}

1. Ferlay J, Soerjomataram I, Dikshit R et al. Cancer incidence and mortality worldwide: sources, methods and major patterns in GLOBOCAN 2012. Int J Cancer 2015; 136(1): E359-86.

2. Gupta A, Shridhar K, Dhillon PK. A review of breast cancer awareness among women in India: cancer literate or awareness deficit? Eur J Cancer 2015; 51: 2058-66.

3. Porter PL. Global trends in breast cancer incidence and mortality. Salud Pu'blica de México 2009; 51 (3): s141-s46.

4. Babu GR, Lakshmi SB, Thiyagarajan JA. Epidemiological correlates of breast cancer in South India. Asian Pac J Cancer Prev 2013; 14 (5): 5077-83.

5. Ali I, Wani WA, Saleem K. Cancer scenario in India 
with future perspectives. Cancer Therapy 2011; 8 (4): 56-70.

6. Srinath Reddy K, Shah B, Varghese C, Ramadoss A. Responding to the threat of chronic diseases in India. Lancet 2005; 366 (6): 1744-9.

7. Balasubramaniam S, Rotti S, Vivekanandam S. Risk factors of female breast carcinoma: a case control study at Puducherry. Indian J Cancer 2013; 50 (4): 65-70.

8. Shreshtha MALVIA, Sarangadhara Appalaraju BAGADI, Uma S. DUBEY and Sunita SAXENA. Epidemiology of breast cancer in Indian women. AsiaPacific Journal of Clinical Oncology 2017; 13 (2): 289295.

9. Tavassoli FA, Devilee P. World Health Organization Classification of Tumors. Tumor of the Breast and Females Genital Organs. Lyon: IARC Press.

10. American College of Radiology Breast Imaging Reporting and Data System (BI-RADS), Ultrasound, 4th edn. Reston, VA: ACR. http:/www.acr.org/ Secondary MainMenu Categories/quality_safety/ BIRADSAtlas/BIRADSAtlasexcerpted text/BIR ADSUItrasoundFirstEdition/ACRBIRADSUSLexi con ClassificationFormDoc1.aspx.

11. Tsunoda HS, Tohno E, Ueno E. Examination of effectiveness of breast cancer detection by modalities and age groups. J Jpn Assoc Breast Cancer Screen 1998;7 (3):281-5.

12. Osako T, Takahashi K, Iwase T, Iijima K, Miyagi Y, Nishimura $\mathrm{S}$, et al. Diagnostic ultrasonography and mammography for invasive and noninvasive breast cancer in women aged 30 to 39 years. Breast Cancer 2007;14 (5):229-33.

13. Kentaro Tamaki, Hironobu Sasano, Takanori Ishida, Kazuyuki Ishida. The Correlation Between Ultrasonographic Findings and Pathologic Features in Breast Disorders. Jpn J Clin Oncol 2010;40(10)905912.

14. Rosen PP. Rosen's Breast Pathology, 3rd edn. Philadelphia, PA: Lippincott Williams \& Wilkins, 2009.

15. Japan Association of Breast and Thyroid Sonology. Guideline for Breast Ultrasound-Management and Diagnosis, 2nd edn. Tokyo: Japanese 2008.

16. Koushi N. Basis of Ultrasound Diagnostic System and Technology. Tokyo: Japanese 2009.

17. Chao TC, Lo YF, Chen SC, Chen MF. Prospective sonographic study of 3039 breast tumors. J Ultrasound Med 1999;18 (4):363-70.

18. Leucht WJ, Rade DR, Humbert KD. Diagnostic value of different interpretative criteria in real-time sonography of the breast. Ultrasound Med Biol 1988;14 (2):59-73.

19. Jackson VP. Sonography of malignant breast disease. Semin Ultrasound CT MR 1989;10 (6):119-31.

Source of Support: Nil; Conflict of Interest: None

Submitted: 13-04-2018; Accepted: 10-05-2018; Published online: 19-05-2018 